\title{
Avelumab first-line maintenance plus best supportive care (BSC) vs BSC alone for advanced urothelial carcinoma: JAVELIN Bladder 100 Japanese subgroup analysis
}

\author{
Yoshihiko Tomita ${ }^{1} \cdot$ Yoshiaki Yamamoto $^{2} \cdot$ Norihiko Tsuchiya $^{3} \cdot$ Hiroomi Kanayama $^{4} \cdot$ Masatoshi Eto $^{5}$. \\ Hideaki Miyake ${ }^{6} \cdot$ Thomas Powles $^{7} \cdot$ Mizuki Yoshida $^{8} \cdot$ Yuichiro Koide $^{8} \cdot$ Yoshiko Umeyama $^{8} \cdot$ Alessandra di Pietro $^{9}$. \\ Hirotsugu Uemura ${ }^{10}$
}

Received: 20 September 2021 / Accepted: 30 October 2021 / Published online: 1 January 2022

(c) The Author(s) 2021

\begin{abstract}
Background The phase 3 JAVELIN Bladder 100 trial showed significantly prolonged overall survival (OS) with avelumab as first-line (1L) maintenance therapy + best supportive care (BSC) vs BSC alone in patients with advanced urothelial carcinoma (UC) that had not progressed with 1L platinum-containing chemotherapy. Efficacy and safety were assessed in patients enrolled in Japan.

Methods Patients with locally advanced or metastatic UC that had not progressed with 4-6 cycles of $1 \mathrm{~L}$ platinum-containing chemotherapy were randomized to avelumab (10 mg/kg intravenously every 2 weeks $)+$ BSC or BSC alone. The primary endpoint was OS, and secondary endpoints included progression-free survival (PFS) and safety.

Results In Japanese patients $(n=73)$ randomized to avelumab + BSC $(n=36)$ or BSC alone $(n=37)$, median OS was 24.7 months (95\% CI, 18.2-not estimable) vs 18.7 months (95\% CI, 12.8-33.0), respectively (HR, 0.81 [95\% CI, $0.41-$ 1.58]), and median PFS was 5.6 months (95\% CI, 1.9-9.4) vs 1.9 months (95\% CI, 1.9-3.8), respectively (HR, 0.63 [95\% $\mathrm{CI}, 0.36-1.11]$ ). In the avelumab $+\mathrm{BSC}$ and BSC-alone arms, grade $\geq 3$ treatment-emergent adverse events (AEs) occurred in $50.0 \%$ vs $8.1 \%$, including grade $\geq 3$ treatment-related AEs in $13.9 \%$ vs $0 \%$, respectively. Efficacy and safety results in Japanese patients were generally consistent with findings in the overall trial population.

Conclusion Avelumab 1L maintenance treatment showed a favorable benefit-risk balance in Japanese patients, supporting avelumab 1L maintenance as a new standard of care in Japanese patients with advanced UC that has not progressed with $1 \mathrm{~L}$ platinum-containing chemotherapy.
\end{abstract}

Trial registration Clinicaltrials.gov NCT02603432.

Keywords Avelumab $\cdot$ Immunotherapy $\cdot$ Maintenance $\cdot$ Japan $\cdot$ Urinary bladder neoplasms

\section{Introduction}

Urothelial carcinoma (UC), which originates in the cells lining the bladder or other parts of the urothelial tract, is one of the most common cancers; bladder cancer itself is the 11th most common cancer globally and the 13th most common cancer in Japan [1,2]. Platinum-based chemotherapy is a standard first-line (1L) treatment for eligible patients with advanced UC $[3,4]$. Although most patients $(\approx 75 \%)$

Yoshihiko Tomita

ytomita@med.niigata-u.ac.jp

Extended author information available on the last page of the article will respond or have disease control initially, long-term benefits are limited and overall survival (OS) is generally short (median, $\approx 9-14$ months) [5-7].

Immune checkpoint inhibitors that target programmed death 1 (PD-1) or programmed death ligand 1 (PD-L1) have shown clinical activity in patients with advanced UC [3]. Consequently, several immune checkpoint inhibitors have been approved in various countries for the treatment of patients with advanced UC that has progressed during or following platinum-containing chemotherapy and in some countries for $1 \mathrm{~L}$ treatment of cisplatin-ineligible patients with PD-L1 + tumors [3, 8]. Avelumab is an anti-PD-L1 antibody [9-11]. In Japan, avelumab has been approved as monotherapy for patients with Merkel cell carcinoma. It has 
also been shown that the combination of avelumab plus axitinib is efficacious and tolerable in Japanese patients with treatment-naive advanced renal cell carcinoma [12], resulting in approval in this indication in Japan.

The phase 3 JAVELIN Bladder 100 trial investigated avelumab as $1 \mathrm{~L}$ maintenance therapy in patients with locally advanced or metastatic UC that had not progressed with $1 \mathrm{~L}$ platinum-containing chemotherapy [13]. The trial met its primary objective, demonstrating significantly prolonged OS with avelumab + best supportive care (BSC) vs BSC alone in all randomized patients (hazard ratio [HR], 0.69 [95\% CI, 0.56-0.86]; $P=0.001)$ and patients with PD-L1 + tumors (HR, 0.56 [95\% CI, 0.40-0.79]; $P<0.001$ ). Results from the trial supported the approval of avelumab $1 \mathrm{~L}$ maintenance in several countries, including in Japan, as well as its inclusion in international treatment guidelines as a new standard of care for advanced UC in cisplatin-eligible and cisplatin-ineligible patients $[3,14,15]$, including guidelines developed by the Japanese Urological Association [16]. Here, we report a post hoc subgroup analysis of the JAVELIN Bladder 100 trial in patients enrolled in Japan.

\section{Materials and methods}

\section{Study design and patients}

JAVELIN Bladder 100 (NCT02603432) was an international, open-label, phase 3 trial. The study design has been reported in detail previously [13]. Briefly, the study enrolled patients who had histologically confirmed, unresectable locally advanced or metastatic UC; had no disease progression after receiving 4-6 cycles of 1L chemotherapy with cisplatin + gemcitabine or carboplatin + gemcitabine; had a treatment-free interval of 4-10 weeks since the last dose of chemotherapy; were aged $\geq 18$ years ( $\geq 20$ years in Japan); and had an ECOG performance status (ECOG PS) of 0 or 1.

Patients were randomly assigned (1:1) to receive either maintenance therapy with avelumab plus BSC (avelumab arm) or BSC alone (control arm). Allocation was stratified by best response to $1 \mathrm{~L}$ chemotherapy (complete response [CR] or partial response [PR] vs stable disease) and by metastatic site when $1 \mathrm{~L}$ chemotherapy was initiated (visceral vs non-visceral). The non-visceral stratum included both patients with unresectable locally advanced disease or only non-visceral disease, including bone metastasis.

The study was conducted in accordance with the ethics principles of the Declaration of Helsinki and Good Clinical Practice guidelines, defined by the International Council for Harmonisation. The protocol, amendments, and informed consent forms were approved by the institutional review board or independent ethics committee at each trial site, and all patients provided written consent.

\section{Outcomes}

The primary endpoint was OS, assessed both in the overall population (all randomized patients) and in patients with PD-L1 + tumors (PD-L1 + population). Secondary endpoints included: progression-free survival (PFS; time from randomization to the date of the first documentation of progressive disease [PD] or death due to any cause, whichever occurs first); objective response (confirmed CR or PR); time to response (time from randomization to the first documentation of objective response) and duration of response (time from the first documentation of objective response to the first documentation of PD or death due to any cause, whichever occurs first) in patients with the objective response; disease control (CR, PR, non-CR/non-PD, or stable disease for $\geq 6$ weeks); and safety. All endpoints were measured after randomization (after chemotherapy), and all tumor assessments were performed according to RECIST version 1.1 with a blinded independent central review (BICR).

\section{Treatment and assessments}

Patients in the avelumab arm were treated with avelumab $10 \mathrm{mg} / \mathrm{kg}$ as a $1-\mathrm{h}$ intravenous infusion every 2 weeks, and all patients also received BSC; patients in the control arm received BSC alone. BSC (e.g., antibiotics, nutritional support, hydration, or pain management) was administered per local practice based on patient needs and clinical judgment; other systemic antitumor therapy was not permitted, but palliative local radiotherapy for isolated lesions was acceptable.

Tumor assessments were performed according to RECIST version 1.1 every 8 weeks for 12 months, then every 12 weeks thereafter, until disease progression was confirmed by BICR. Adverse events (AEs) were graded according to the National Cancer Institute Common Terminology Criteria for AEs, version 4.03. PD-L1 expression was assessed in tumor samples using the Ventana PD-L1 immunohistochemistry assay (SP263; Ventana Medical Systems). PD-L1 + status was defined as $\geq 1$ of the following: $\geq 25 \%$ of tumor cells stained for PD-L1, $\geq 25 \%$ of immune cells stained for PD-L1 if $>1 \%$ of the tumor area contained immune cells, or $100 \%$ of immune cells stained for PD-L1 if $\leq 1 \%$ of the tumor area contained immune cells.

\section{Statistical analysis}

The Japanese subgroup comprised all randomized patients enrolled at sites in Japan. Efficacy endpoints were assessed 
for the Japanese subgroup according to the intentionto-treat principle. OS and PFS were estimated using the Kaplan-Meier method, and HRs and associated 95\% CIs for OS and PFS were calculated using an unstratified Cox proportional hazards model. Objective response rate and disease control rate were calculated by treatment group, and exact 2-sided 95\% CIs were calculated using the Clopper-Pearson method. Safety was assessed in all patients who received $\geq 1$ dose of avelumab in the avelumab arm and in all the patients who completed the cycle 1 day 1 visit in the control arm. AEs were summarized using Medical Dictionary for Regulatory Activities (version 22.1) preferred terms. Statistical analyses were conducted using SAS, version 9.4. Data from a preplanned interim analysis are reported; however, because the primary endpoints were met, this represents the final analysis. The data cutoff date for all analyses was October 21, 2019.

\section{Results}

\section{Patient characteristics and disposition}

Details of all patients enrolled in the JAVELIN Bladder 100 trial have been reported previously [13]. Overall, 700 patients were randomized to receive avelumab maintenance therapy + BSC (avelumab arm; $n=350$ ) or BSC alone (control arm; $n=350$ ). In total, 73 patients were enrolled in Japan; 36 and 37 were randomized to the avelumab and control arms, respectively. In the overall population, $51.1 \%$ of patients had a PD-L1 + tumor, including $57.5 \%$ of patients in the Japanese subgroup.

Demographics and baseline characteristics were generally balanced between the treatment arms (Table 1). Differences noted between the Japanese subgroup and the overall population included (avelumab/control arm): lower median weight (62.9/61.9 kg vs 72.4/73.0 kg), lower proportion with an ECOG PS of $\geq 1(16.7 \% / 10.8 \%$ vs $39.1 \% / 39.7 \%)$, higher proportion with an upper tract primary tumor $(58.3 \% / 56.8 \%$ vs $30.3 \% / 23.1 \%$ ), and slightly higher proportion with baseline creatinine clearance $<60 \mathrm{~mL} / \mathrm{min}(58.3 \% / 51.4 \%$ vs $48.0 \% / 42.3 \%$ ). Furthermore, in Japanese patients with PD-L1 + tumors, differences in demographics and baseline characteristics between the avelumab and control arms included: higher proportion with an ECOG PS of $\geq 1$ (21.1\% vs $4.3 \%$; Table 1$)$, lower proportion with visceral metastases ( $26.3 \%$ vs $47.8 \%$; Table 1), higher proportion with baseline creatinine clearance $<60 \mathrm{~mL} / \mathrm{min}(63.2 \%$ vs $39.1 \%$; Table 1$)$, and lower proportion who had an objective response to $1 \mathrm{~L}$ chemotherapy (52.6\% vs $65.2 \%$; Table 2 ).

Differences in prior $1 \mathrm{~L}$ chemotherapy were observed between the Japanese subgroup and the overall population (Table 2), including (avelumab/control arm): higher proportion who had received $1 \mathrm{~L}$ gemcitabine + cisplatin (69.4\%/78.4\% vs $52.3 \% / 58.9 \%)$ and lower proportion who had an objective response to $1 \mathrm{~L}$ chemotherapy $(61.1 \% / 59.5 \%$ vs $72.3 \% / 72.0 \%$ ). Median durations of $1 \mathrm{~L}$ chemotherapy in the Japanese subgroup and overall population in the avelumab/control arm were $16.7 / 18.0$ vs $18.0 / 18.0$ weeks for cisplatin, 15.6/15.6 vs 17.0/16.1 weeks for carboplatin, and $17.3 / 18.9$ vs $19.0 / 19.0$ weeks for gemcitabine.

At the data cutoff date, 7 of 36 (19.4\%) Japanese patients in the avelumab arm and 2 of $37(5.4 \%)$ in the control arm remained on study treatment (Online Resource 1). Of patients who had discontinued from the avelumab arm (29 [80.6\%]) or control arm (35 [94.6\%]), reasons for treatment discontinuation were: disease progression (21 [58.3\%] vs 28 [75.7\%]), AE (4 [11.1\%] vs 0), death (2 [5.6\%] vs 0), withdrawal by patient ( $1[2.8 \%]$ vs $6[16.2 \%])$, physician's decision (1 [2.8\%] vs 0$)$, and overall health deterioration (0 vs $1[2.7 \%])$.

\section{Efficacy}

Efficacy results in the Japanese subgroup were generally consistent with those in the overall population. Median follow-up for OS in the Japanese subgroup was 24.2 months (95\% CI, 18.8-31.8) in the avelumab arm vs 24.1 months (95\% CI, 17.8-28.1) in the control arm. OS in both the Japanese subgroup and overall population are shown in Fig. 1. In the Japanese subgroup, median OS was 24.7 months (95\% CI, 18.2-not estimable) in the avelumab arm vs 18.7 months (95\% CI, 12.8-33.0) in the control arm (HR, 0.81 [95\% CI, 0.41-1.58]) (Fig. 1A). In Japanese patients with PD-L1 + tumors, median OS was 18.6 months $(95 \%$ CI, 9.4-not estimable) in the avelumab arm vs 19.4 months (95\% CI, 11.7-33.0) in the control arm (HR, 1.00 [95\% CI, 0.41-2.41]) (Fig. 1C).

PFS in both the Japanese subgroup and overall population are shown in Fig. 2. Median PFS (by BICR) in Japanese patients was 5.6 months (95\% CI, 1.9-9.4) in the avelumab arm vs 1.9 months (95\% CI, 1.9-3.8) in the control arm (HR, 0.63 [95\% CI, 0.36-1.11]) (Fig. 2A). In Japanese patients with PD-L1 + tumors, median PFS was 5.6 months $(95 \%$ CI, 1.8-11.2) in the avelumab arm vs 1.9 months (95\% CI, 1.9-3.8) in the control arm (HR, 0.62 [95\% CI, 0.30-1.30]) (Fig. 2C).

The objective response rates (by BICR) in the Japanese subgroup in the avelumab and control arms were 5.6\% (95\% CI, 0.7-18.7) vs 0\% (95\% CI, 0-9.5), respectively (Table 3), including $1 \mathrm{CR}$ and $1 \mathrm{PR}$ in the avelumab arm. In the 2 responding patients, time to response was 2.0 and 5.6 months, and duration of response was 34.0 months (ongoing at data cutoff) and 8.3 months, respectively. 
Table 1 Demographics and baseline characteristics of the Japanese subgroup and the overall population. Source: Data for the overall population have been published previously in Ref. [13]. Copyright (C) 2020 Massachusetts Medical Society. Reprinted with permission

\begin{tabular}{|c|c|c|c|c|c|c|c|c|}
\hline & \multicolumn{4}{|c|}{ Japanese subgroup } & \multicolumn{4}{|c|}{ Overall population [13] } \\
\hline & \multicolumn{2}{|c|}{$\begin{array}{l}\text { Randomised patients } \\
(n=73)\end{array}$} & \multicolumn{2}{|c|}{$\begin{array}{l}\text { PD-L1 + population } \\
(n=42)\end{array}$} & \multicolumn{2}{|c|}{$\begin{array}{l}\text { Randomised patients } \\
(N=700)\end{array}$} & \multicolumn{2}{|c|}{$\begin{array}{l}\text { PD-L1 + population } \\
(N=358)\end{array}$} \\
\hline & $\begin{array}{l}\text { Avelumab } \\
+ \text { BSC }(n=36)\end{array}$ & $\mathrm{BSC}(n=37)$ & $\begin{array}{l}\text { Avelumab } \\
+\mathrm{BSC} \\
(n=19)\end{array}$ & $\mathrm{BSC}(n=23)$ & $\begin{array}{l}\text { Avelumab } \\
+ \text { BSC } \\
(n=350)\end{array}$ & $\begin{array}{l}\text { BSC } \\
(n=350)\end{array}$ & $\begin{array}{l}\text { Avelumab } \\
+ \text { BSC } \\
(n=189)\end{array}$ & $\operatorname{BSC}(n=169)$ \\
\hline $\begin{array}{l}\text { Age, median } \\
\text { (range), years }\end{array}$ & $70.5(46-84)$ & $69(43-83)$ & $71(55-84)$ & $69(49-83)$ & $68(37-90)$ & $69(32-89)$ & $70(37-90)$ & $70(32-84)$ \\
\hline $\begin{array}{l}\text { Weight, } \\
\text { median } \\
\text { (range), kg }\end{array}$ & $\begin{array}{l}62.9(41.9- \\
100.6)\end{array}$ & $\begin{array}{l}61.9 \\
(37.2-86.8)\end{array}$ & $\begin{array}{l}62.8(44.8- \\
100.6)\end{array}$ & $\begin{array}{l}62.8(37.2- \\
86.8)\end{array}$ & $\begin{array}{l}72.4(40.0- \\
136.0)\end{array}$ & $\begin{array}{l}73.0(37.2- \\
135.3)\end{array}$ & $\begin{array}{l}73.5(40.0- \\
136.0)\end{array}$ & $\begin{array}{l}73.5(37.2- \\
131.8)\end{array}$ \\
\hline \multicolumn{9}{|l|}{ Sex, $n(\%)$} \\
\hline Male & $25(69.4)$ & $26(70.3)$ & $12(63.2)$ & $16(69.6)$ & $266(76.0)$ & 275 (78.6) & 145 (76.7) & $129(76.3)$ \\
\hline Female & $11(30.6)$ & $11(29.7)$ & $7(36.8)$ & $7(30.4)$ & $84(24.0)$ & $75(21.4)$ & $44(23.3)$ & $40(23.7)$ \\
\hline \multicolumn{9}{|l|}{ Race, $n(\%)$} \\
\hline White & 0 & 0 & 0 & 0 & $232(66.3)$ & $238(68.0)$ & $121(64.0)$ & $119(70.4)$ \\
\hline Asian & $36(100)$ & $37(100)$ & $19(100)$ & $23(100)$ & $75(21.4)$ & $81(23.1)$ & $42(22.2)$ & $33(19.5)$ \\
\hline $\begin{array}{l}\text { Black/ } \\
\text { African } \\
\text { American }\end{array}$ & 0 & 0 & 0 & 0 & $2(0.6)$ & 0 & $1(0.5)$ & 0 \\
\hline Other & 0 & 0 & 0 & 0 & $21(6.0)$ & $15(4.3)$ & $12(6.3)$ & $7(4.1)$ \\
\hline Unknown & 0 & 0 & 0 & 0 & $20(5.7)$ & $16(4.6)$ & $13(6.9)$ & $10(5.9)$ \\
\hline \multicolumn{9}{|l|}{$\begin{array}{l}\text { Pooled } \\
\text { geographic } \\
\text { region, } n(\%)\end{array}$} \\
\hline $\begin{array}{l}\text { North } \\
\text { America }\end{array}$ & 0 & 0 & 0 & 0 & $12(3.4)$ & $22(6.3)$ & $8(4.2)$ & $8(4.7)$ \\
\hline Europe & 0 & 0 & 0 & 0 & $214(61.1)$ & $203(58.0)$ & $110(58.2)$ & $102(60.4)$ \\
\hline Asia & $36(100)$ & $37(100)$ & $19(100)$ & $23(100)$ & 73 (20.9) & $74(21.1)$ & $40(21.2)$ & $31(18.3)$ \\
\hline Australasia & 0 & 0 & 0 & 0 & $34(9.7)$ & $37(10.6)$ & $20(10.6)$ & $24(14.2)$ \\
\hline $\begin{array}{l}\text { Rest of the } \\
\text { world }\end{array}$ & 0 & 0 & 0 & 0 & $17(4.9)$ & $14(4.0)$ & $11(5.8)$ & $4(2.4)$ \\
\hline \multicolumn{9}{|l|}{$\begin{array}{l}\text { ECOG perfor- } \\
\text { mance status, } \\
n(\%)\end{array}$} \\
\hline 0 & $30(83.3)$ & 33 (89.2) & $15(78.9)$ & $22(95.7)$ & $213(60.9)$ & $211(60.3)$ & $114(60.3)$ & $107(63.3)$ \\
\hline 1 & $5(13.9)$ & $4(10.8)$ & $3(15.8)$ & $1(4.3)$ & $136(38.9)$ & 136 (38.9) & $74(39.2)$ & $61(36.1)$ \\
\hline 2 & $1(2.8)$ & 0 & $1(5.3)$ & 0 & $1(0.3)$ & 0 & $1(0.5)$ & 0 \\
\hline 3 & 0 & 0 & 0 & 0 & 0 & $3(0.9)$ & 0 & $1(0.6)$ \\
\hline \multicolumn{9}{|l|}{$\begin{array}{r}\text { Site of primary } \\
\text { tumor, } n(\%)^{\mathrm{a}}\end{array}$} \\
\hline Upper tract & $21(58.3)$ & $21(56.8)$ & $10(52.6)$ & $13(56.5)$ & $106(30.3)$ & $81(23.1)$ & $44(23.3)$ & $35(20.7)$ \\
\hline Lower tract & $15(41.7)$ & $16(43.2)$ & $9(47.4)$ & $10(43.5)$ & $244(69.7)$ & 269 (76.9) & 145 (76.7) & $134(79.3)$ \\
\hline \multicolumn{9}{|l|}{$\begin{array}{l}\text { Site of baseline } \\
\text { metastasis } \\
\text { before chem- } \\
\text { otherapy, } n \\
(\%)\end{array}$} \\
\hline Visceral & $17(47.2)$ & $19(51.4)$ & $5(26.3)$ & $11(47.8)$ & $191(54.6)$ & $191(54.6)$ & 88 (46.6) & 79 (46.7) \\
\hline Non-visceral $^{\mathrm{b}}$ & $19(52.8)$ & $18(48.6)$ & $14(73.7)$ & $12(52.2)$ & $159(45.4)$ & $159(45.4)$ & $101(53.4)$ & $90(53.3)$ \\
\hline $\begin{array}{l}\text { Creatinine } \\
\text { clearance, } n \\
(\%)\end{array}$ & & & & & & & & \\
\hline
\end{tabular}


Table 1 (continued)

\begin{tabular}{|c|c|c|c|c|c|c|c|c|}
\hline & \multicolumn{4}{|c|}{ Japanese subgroup } & \multicolumn{4}{|c|}{ Overall population [13] } \\
\hline & \multicolumn{2}{|c|}{$\begin{array}{l}\text { Randomised patients } \\
(n=73)\end{array}$} & \multicolumn{2}{|c|}{$\begin{array}{l}\text { PD-L1 + population } \\
(n=42)\end{array}$} & \multicolumn{2}{|c|}{$\begin{array}{l}\text { Randomised patients } \\
(N=700)\end{array}$} & \multicolumn{2}{|c|}{$\begin{array}{l}\text { PD-L1 + population } \\
(N=358)\end{array}$} \\
\hline & $\begin{array}{l}\text { Avelumab } \\
+ \text { BSC }(n=36)\end{array}$ & $\mathrm{BSC}(n=37)$ & $\begin{array}{l}\text { Avelumab } \\
+\mathrm{BSC} \\
(n=19)\end{array}$ & $\operatorname{BSC}(n=23)$ & $\begin{array}{l}\text { Avelumab } \\
+\mathrm{BSC} \\
(n=350)\end{array}$ & $\begin{array}{l}\text { BSC } \\
(n=350)\end{array}$ & $\begin{array}{l}\text { Avelumab } \\
+\mathrm{BSC} \\
(n=189)\end{array}$ & $\operatorname{BSC}(n=169)$ \\
\hline$\geq 60 \mathrm{~mL} / \mathrm{min}$ & $15(41.7)$ & $16(43.2)$ & $7(36.8)$ & $12(52.2)$ & $181(51.7)$ & $196(56.0)$ & $104(55.0)$ & $97(57.4)$ \\
\hline$<60 \mathrm{~mL} / \mathrm{min}$ & $21(58.3)$ & $19(51.4)$ & $12(63.2)$ & $9(39.1)$ & $168(48.0)$ & $148(42.3)$ & $84(44.4)$ & $70(41.4)$ \\
\hline Unknown & 0 & $2(5.4)$ & 0 & $2(8.7)$ & $1(0.3)$ & $6(1.7)$ & $1(0.5)$ & $2(1.2)$ \\
\hline \multicolumn{9}{|l|}{$\begin{array}{l}\text { PD-L1 status, } \\
n(\%)\end{array}$} \\
\hline Positive & $19(52.8)$ & $23(62.2)$ & $19(100)$ & $23(100)$ & $189(54.0)$ & $169(48.3)$ & $189(100)$ & $169(100)$ \\
\hline Negative & $15(41.7)$ & $9(24.3)$ & 0 & 0 & $139(39.7)$ & $131(37.4)$ & 0 & 0 \\
\hline Unknown & $2(5.6)$ & $5(13.5)$ & 0 & 0 & $22(6.3)$ & $50(14.3)$ & 0 & 0 \\
\hline
\end{tabular}

$B S C$ best supportive care, $P D-L 1$ programmed death ligand 1

${ }^{a}$ The upper tract was defined as the renal pelvis or ureter; the lower tract was defined as the bladder, urethra, or prostate gland

${ }^{b}$ Non-visceral includes patients with locally advanced disease or only non-visceral disease, including bone metastasis

\section{Subsequent therapy}

Compared with the overall population, a higher proportion of Japanese patients received a subsequent anticancer drug therapy after discontinuing study therapy (Table 4). In Japanese patients in the avelumab vs control arms, a subsequent anticancer drug therapy was received by $61.1 \%$ vs $81.1 \%$, respectively (compared with $42.3 \%$ vs $61.7 \%$, respectively, in the overall population). This included a PD-1 or PD-L1 inhibitor in $38.9 \%$ vs $67.6 \%$ of the avelumab vs control arms, respectively (compared with $6.3 \%$ vs $43.7 \%$, respectively, in the overall population). In the Japanese subgroup, the most commonly received subsequent anticancer drug therapies (avelumab vs control arm, respectively) were pembrolizumab (38.9\% vs $64.9 \%)$, gemcitabine ( $36.1 \%$ vs $43.2 \%$ ), carboplatin (30.6\% vs $21.6 \%$ ), cisplatin ( $25.0 \%$ vs $29.7 \%$ ), and paclitaxel (13.9\% vs 16.2\%) (Online Resource 2).

\section{Safety}

At data cutoff, the median duration of treatment in Japanese patients was 32.0 weeks (range, 2.0-159.9) in the avelumab arm and 9.1 weeks (range, 0.1-90.0) in the control arm (Online Resource 3). The median avelumab dose intensity was $17.6 \mathrm{mg} / \mathrm{kg} / 4$-week cycle (range, 10.0-19.8), with a median relative dose intensity of $87.8 \%$ (range, 50.0-99.1), similar to that in the overall population.

The safety profile of avelumab in Japanese patients was generally consistent with that in the overall population (Table 5), with slight differences in rates of some treatmentemergent AEs (TEAEs; related or unrelated to treatment) (Table 6). TEAEs of any grade occurred in all Japanese patients in the avelumab arm and in $56.8 \%$ of patients in the control arm, including grade $\geq 3$ TEAEs in $50.0 \%$ and $8.1 \%$ of patients, respectively. In the avelumab arm, the most common TEAEs of any grade were pyrexia $(27.8 \%)$, anemia (19.4\%), and nasopharyngitis (19.4\%); the most common grade $\geq 3$ TEAEs were anemia (11.1\%), pyelonephritis (5.6\%), increased amylase (5.6\%), and increased blood triglycerides (5.6\%). TEAEs led to interruption of avelumab in 16 patients (44.4\%; most commonly due to pyelonephritis in 3 patients [8.3\%], hyperthyroidism in 2 patients [5.6\%]) and discontinuation of avelumab in 4 patients $(11.1 \%$; 1 case each due to anemia, myocardial infarction, gastric ulcer, sepsis, platelet count decreased, and interstitial lung disease).

In avelumab-treated patients, slight differences were seen in the most common TEAEs of any grade in Japanese patients compared with the overall population, including an increased occurrence of pyrexia (27.8\% vs $14.8 \%)$ and nasopharyngitis (19.4\% vs $7.6 \%$ ) and a lower occurrence of fatigue ( $5.6 \%$ vs $17.7 \%)$, decreased appetite $(2.8 \%$ vs $13.7 \%$ ), and asthenia (0\% vs $16.3 \%$ ) (Table 6). The rate of discontinuation due to TEAEs in avelumab-treated patients was similar between the Japanese subgroup and the overall population (11.1\% vs $11.9 \%)$.

Treatment-related AEs (TRAEs) of any grade with avelumab occurred in $75.0 \%$ of patients in the Japanese subgroup, including grade $\geq 3$ TRAEs in $13.9 \%$ of patients (Online Resource 4). The most common TRAEs of any grade were hypothyroidism (16.7\%), pyrexia (16.7\%), infusion-related reaction (single term; 13.9\%), hyperthyroidism (11.1\%), and stomatitis (11.1\%); the most common grade $\geq 3$ TRAE was anemia (5.6\%). 
Table 2 Summary of first-line chemotherapy received by patients in the Japanese subgroup and the overall population. Source: Data for the overall population have been published previously in Ref. [13]. Copyright ( 2020 Massachusetts Medical Society. Reprinted with permission

\begin{tabular}{|c|c|c|c|c|c|c|c|c|}
\hline & \multicolumn{4}{|c|}{ Japanese subgroup } & \multicolumn{4}{|c|}{ Overall population [13] } \\
\hline & \multicolumn{2}{|c|}{$\begin{array}{l}\text { Randomized patients } \\
(n=73)\end{array}$} & \multicolumn{2}{|c|}{$\begin{array}{l}\text { PD-L1 + population } \\
(n=42)\end{array}$} & \multicolumn{2}{|c|}{$\begin{array}{l}\text { Randomized patients } \\
(N=700)\end{array}$} & \multicolumn{2}{|c|}{$\begin{array}{l}\text { PD-L1 + population } \\
(N=358)\end{array}$} \\
\hline & $\begin{array}{l}\text { Avelumab } \\
+ \text { BSC } \\
(n=36)\end{array}$ & $\begin{array}{l}\mathrm{BSC} \\
(n=37)\end{array}$ & $\begin{array}{l}\text { Avelumab } \\
+ \text { BSC } \\
(n=19)\end{array}$ & $\begin{array}{l}\mathrm{BSC} \\
(n=23)\end{array}$ & $\begin{array}{l}\text { Avelumab } \\
+ \text { BSC } \\
(n=350)\end{array}$ & $\begin{array}{l}\mathrm{BSC} \\
(n=350)\end{array}$ & $\begin{array}{l}\text { Avelumab } \\
+ \text { BSC } \\
(n=189)\end{array}$ & $\begin{array}{l}\text { BSC } \\
(n=169)\end{array}$ \\
\hline \multicolumn{9}{|l|}{$\begin{array}{l}1 \mathrm{~L} \text { chemotherapy } \\
\text { regimen, } n(\%)\end{array}$} \\
\hline $\begin{array}{l}\text { Gemcitabine + } \\
\text { cisplatin }\end{array}$ & $25(69.4)$ & $29(78.4)$ & $14(73.7)$ & $18(78.3)$ & $183(52.3)$ & $206(58.9)$ & $101(53.4)$ & $98(58.0)$ \\
\hline $\begin{array}{l}\text { Gemcitabine + } \\
\text { carboplatin }\end{array}$ & $9(25.0)$ & $8(21.6)$ & $4(21.1)$ & $5(21.7)$ & $147(42.0)$ & $122(34.9)$ & $74(39.2)$ & $54(32.0)$ \\
\hline $\begin{array}{c}\text { Gemcitabine }+ \\
\text { cisplatin or } \\
\text { carboplatin }^{\mathrm{a}}\end{array}$ & $2(5.6)$ & 0 & $1(5.3)$ & 0 & $20(5.7)$ & $20(5.7)$ & $14(7.4)$ & $15(8.9)$ \\
\hline Not reported & 0 & 0 & 0 & 0 & 0 & $2(0.6)$ & 0 & $2(1.2)$ \\
\hline \multicolumn{9}{|l|}{$\begin{array}{l}\text { Best response to } 1 \mathrm{~L} \\
\text { chemotherapy, } n \\
(\%)\end{array}$} \\
\hline $\begin{array}{l}\text { Complete or partial } \\
\text { response }\end{array}$ & $22(61.1)$ & $22(59.5)$ & $10(52.6)$ & $15(65.2)$ & $253(72.3)$ & $252(72.0)$ & $139(73.5)$ & $128(75.7)$ \\
\hline Stable disease & $14(38.9)$ & $15(40.5)$ & $9(47.4)$ & $8(34.8)$ & $97(27.7)$ & $98(28.0)$ & $50(26.5)$ & $41(24.3)$ \\
\hline \multicolumn{9}{|l|}{$\begin{array}{l}\text { Cisplatin, duration of } \\
\text { treatment }\end{array}$} \\
\hline$n$ & 27 & 29 & 15 & 18 & 203 & 226 & 115 & 113 \\
\hline $\begin{array}{l}\text { Median (range), } \\
\text { weeks }\end{array}$ & $\begin{array}{l}16.7 \\
(3.0-37.6)\end{array}$ & $\begin{array}{l}18.0 \\
(12.0-54.1)\end{array}$ & $\begin{array}{l}15.9 \\
(7.0-37.6)\end{array}$ & $\begin{array}{l}18.9 \\
(12.0-54.1)\end{array}$ & $\begin{array}{l}18.0 \\
(3.0-37.6)\end{array}$ & $\begin{array}{l}18.0 \\
(3.0-82.9)\end{array}$ & $\begin{array}{l}18.0 \\
(3.0-37.6)\end{array}$ & $\begin{array}{l}18.0 \\
(3.0-54.1)\end{array}$ \\
\hline \multicolumn{9}{|l|}{$\begin{array}{l}\text { Carboplatin, duration } \\
\text { of treatment }\end{array}$} \\
\hline$n$ & 11 & 8 & 5 & 5 & 167 & 142 & 88 & 69 \\
\hline $\begin{array}{l}\text { Median (range), } \\
\text { weeks }\end{array}$ & $\begin{array}{l}15.6 \\
(8.9-23.1)\end{array}$ & $\begin{array}{l}15.6 \\
(13.3-18.7)\end{array}$ & $\begin{array}{l}13.9 \\
(12.4-23.1)\end{array}$ & $\begin{array}{l}14.1 \\
(13.3-18.7)\end{array}$ & $\begin{array}{l}17.0 \\
(3.0-39.9)\end{array}$ & $\begin{array}{l}16.1 \\
(3.0-36.1)\end{array}$ & $\begin{array}{l}17.3 \\
(3.0-24.7)\end{array}$ & $\begin{array}{l}14.0 \\
(3.0-22.1)\end{array}$ \\
\hline \multicolumn{9}{|l|}{$\begin{array}{l}\text { Gemcitabine, dura- } \\
\text { tion of treatment }\end{array}$} \\
\hline$n$ & 36 & 37 & 19 & 23 & 350 & 348 & 189 & 167 \\
\hline $\begin{array}{l}\text { Median (range), } \\
\text { weeks }\end{array}$ & $\begin{array}{l}17.3 \\
(10.9-38.4)\end{array}$ & $\begin{array}{l}18.9 \\
(12.6-55.3)\end{array}$ & $\begin{array}{l}17.1 \\
(13.0-38.4)\end{array}$ & $\begin{array}{l}18.9 \\
(12.6-55.3)\end{array}$ & $\begin{array}{l}19.0 \\
(9.0-39.9)\end{array}$ & $\begin{array}{l}19.0 \\
(9.9-82.9)\end{array}$ & $\begin{array}{l}19.0 \\
(9.0-38.4)\end{array}$ & $\begin{array}{l}18.6 \\
(9.9-55.3)\end{array}$ \\
\hline
\end{tabular}

$1 L$ first-line, $B S C$ best supportive care, $P D-L 1$ programmed death ligand 1

${ }^{a}$ Includes patients who switched platinum-based regimens while receiving $1 \mathrm{~L}$ chemotherapy

Using a composite term to identify infusion-related reactions (including several prespecified preferred terms in addition to signs and symptoms of infusion-related reaction), 10 patients $(27.8 \%$ ) had an infusion-related reaction (Table 5); all were grade $1 / 2$.

In the Japanese subgroup, immune-related AEs (irAEs) of any grade occurred in $36.1 \%$ treated with avelumab, including grade 3 irAEs in 8.3\%; no grade 4/5 irAEs occurred during the study (Online Resource 5). The irAEs comprised immune-related rash (19.4\%), thyroid disorders (13.9\%), pneumonitis (5.6\%), and enteritis, adrenal insufficiency, vitiligo, and uveitis, each in $2.8 \%$. Grade 3 irAEs comprised immune-related rash in $5.6 \%$ and enteritis in $2.8 \%$. One Japanese patient (2.8\%) discontinued avelumab because of an irAE (pneumonitis), compared with 5.5\% discontinuing because of irAEs in the overall population.

Serious AEs occurred in 9 patients $(25.0 \%)$ in the avelumab arm and in 2 patients (5.4\%) in the control arm of the Japanese subgroup, including serious TRAEs in 5 patients $(13.9 \%)$ in the avelumab arm (1 case each of gastric ulcer, vomiting, colitis, sepsis, anemia, decreased platelet count, interstitial lung disease, and myocardial infarction; some patients had $\geq 1$ type of serious AE). One patient in the avelumab arm died following a TEAE (classified as treatment 
A

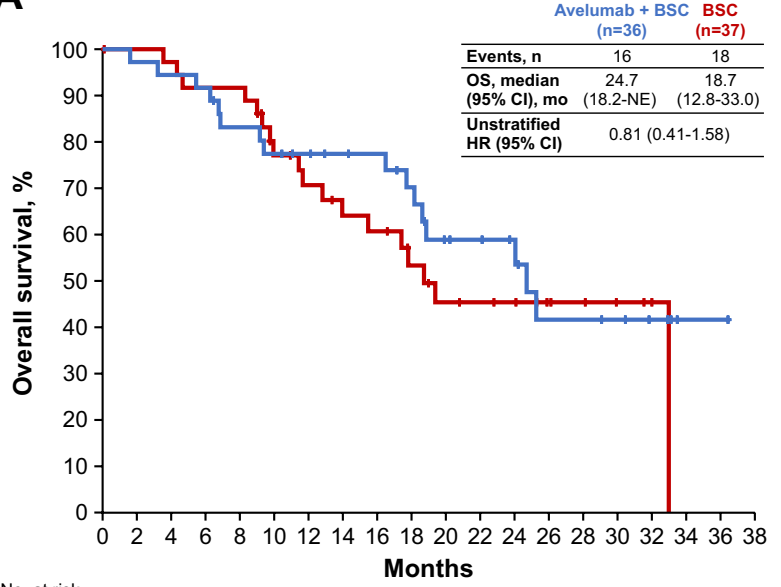
No. at risk

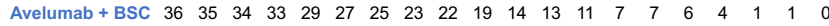
$\begin{array}{lllllllllllllllllllll}\text { BSC } & 37 & 36 & 35 & 33 & 33 & 25 & 22 & 19 & 18 & 14 & 11 & 10 & 9 & 6 & 5 & 3 & 2 & 0 & 0 & 0\end{array}$

C
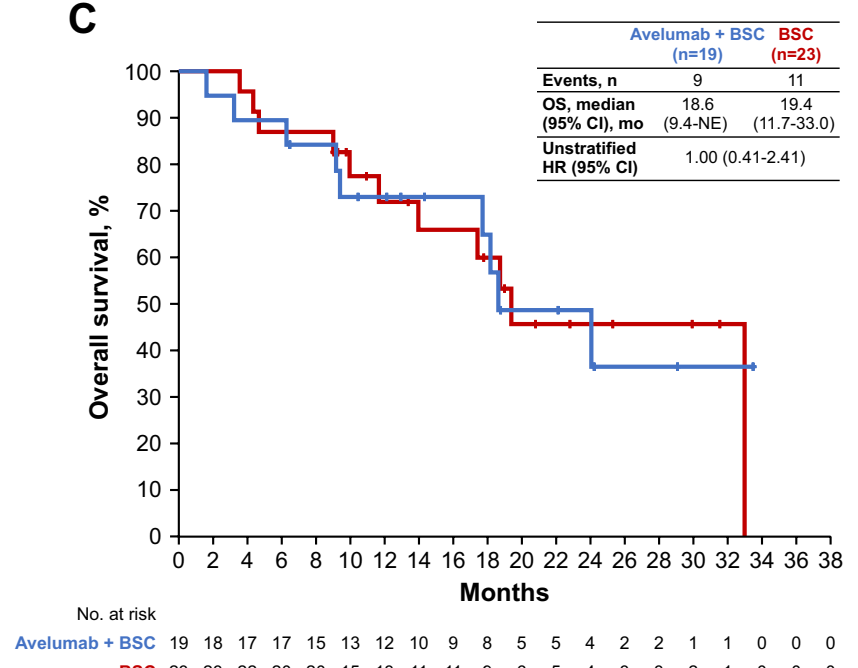

Fig. 1 OS in A the Japanese subgroup, B the overall population [13], C Japanese patients with PD-L1 + tumors, and D all patients with PD-L1 + tumors [13]. BSC best supportive care, $H R$ hazard ratio, $N E$ not estimable, $O S$ overall survival, $P D-L 1$ programmed death ligand

related); the patient had sepsis after a urinary tract infection and possible central venous catheter infection after receiving 11 infusions of avelumab, as reported previously [13].

\section{Discussion}

Overall results from the JAVELIN Bladder 100 trial showed that avelumab $1 \mathrm{~L}$ maintenance $+\mathrm{BSC}$ resulted in significantly longer OS than BSC alone in patients with advanced UC that had not progressed with $1 \mathrm{~L}$ platinumcontaining chemotherapy, both in the overall population and PD-L1 + population [13]. Findings from the Japanese subgroup were generally consistent with those in the

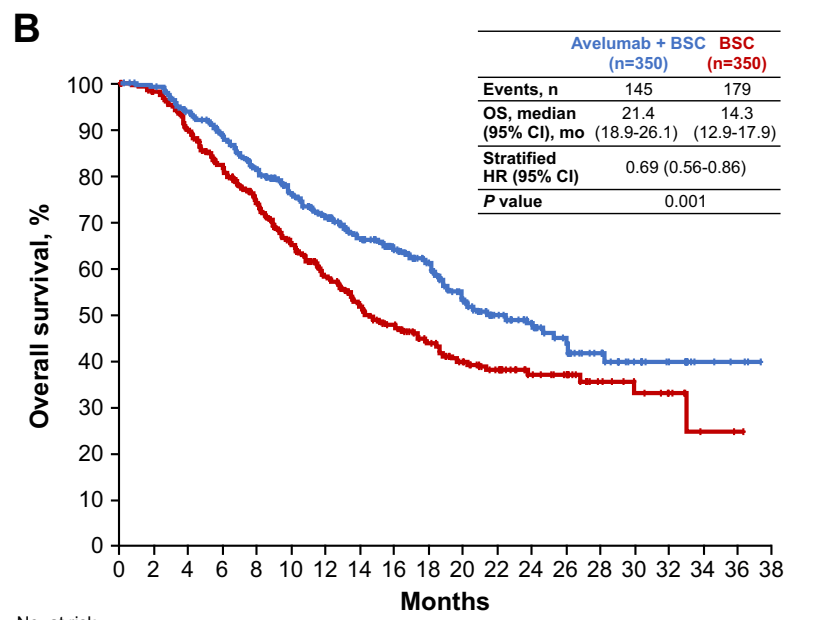
No. at risk

Avelumab + BSC $350342318294259226196167145122 \quad 87 \quad 65 \quad 51 \quad 39 \quad 26 \quad 15 \quad 11 \quad 5 \quad 3 \quad 0$

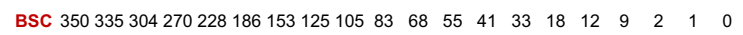

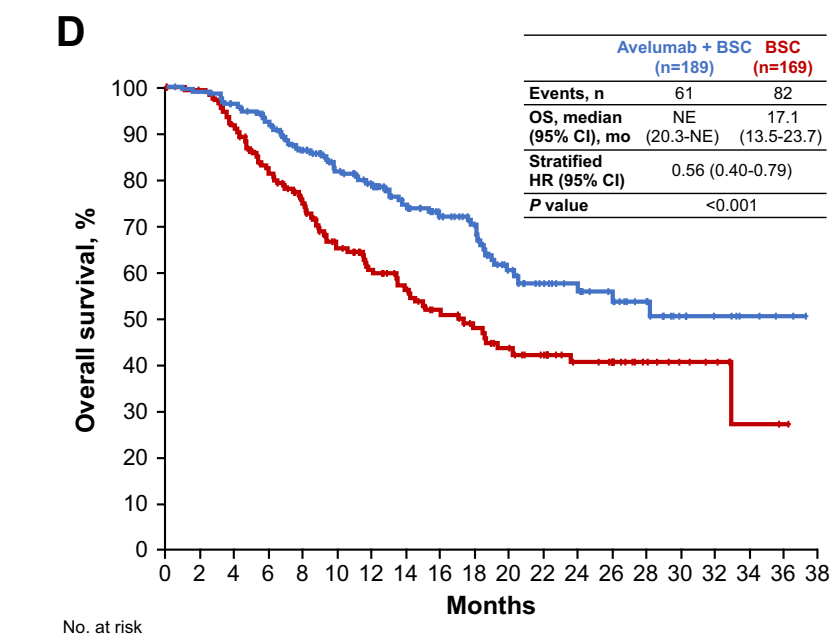

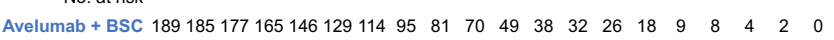

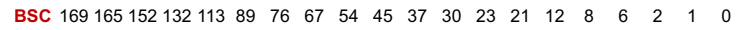

1. At data cutoff (October 21, 2019), the median follow-up for OS in all Japanese patients was $\geq 24$ months in both arms. B and D From Powles et al. [13]. Copyright (C) 2020 Massachusetts Medical Society. Reprinted with permission

overall population within the limitations of an underpowered exploratory subgroup analysis, further demonstrating the efficacy and safety of avelumab 1L maintenance.

Demographic and baseline characteristics in the Japanese subgroup were generally balanced between treatment arms. Minor differences between the total Japanese subgroup and the overall population included lower median weight, lower proportion with an ECOG $\mathrm{PS} \geq 1$, and higher proportion with an upper tract primary tumor or with baseline creatinine clearance $<60 \mathrm{~mL} / \mathrm{min}$. In addition, higher proportion of the Japanese subgroup had received gemcitabine + cisplatin as $1 \mathrm{~L}$ chemotherapy and lower proportion had achieved an objective response with $1 \mathrm{~L}$ chemotherapy. The duration 


\section{A}

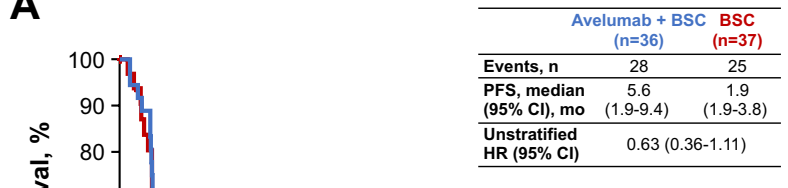

$\begin{array}{lllllllllllllllllllll}\text { Avelumab + BSC } & 36 & 20 & 19 & 16 & 12 & 10 & 7 & 6 & 6 & 5 & 5 & 5 & 4 & 3 & 2 & 2 & 1 & 1 & 0 & 0\end{array}$

BSC $\begin{array}{llllllllllllllllllll}37 & 12 & 7 & 6 & 5 & 3 & 3 & 2 & 2 & 2 & 0 & 0 & 0 & 0 & 0 & 0 & 0 & 0 & 0 & 0\end{array}$

\section{C}

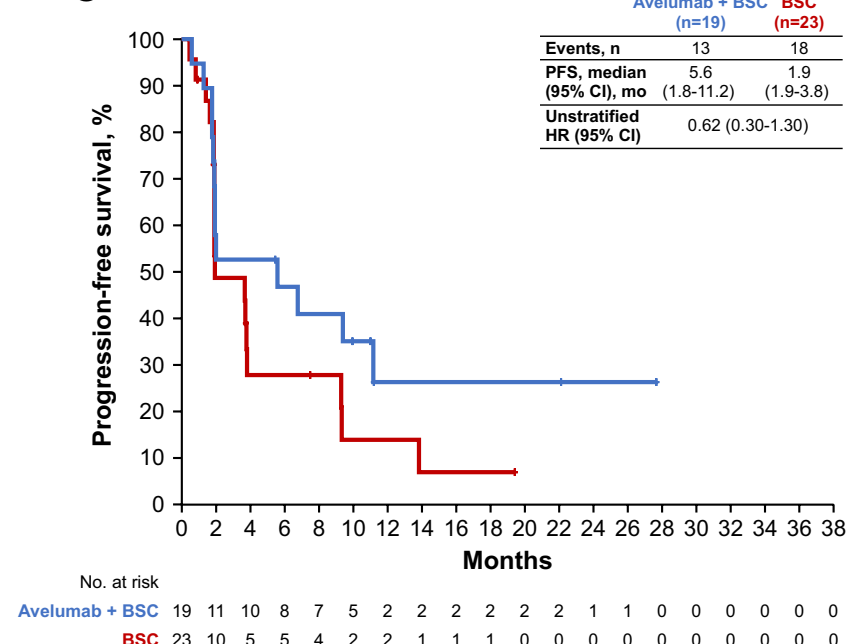

Fig. 2 PFS by BICR in A the Japanese subgroup, B the overall population [13], C Japanese patients with PD-L1 + tumors, and D all patients with PD-L1 + tumors [13]. BICR blinded independent central review, $B S C$ best supportive care, $H R$ hazard ratio, $P D-L 1$ pro-

of prior $1 \mathrm{~L}$ chemotherapy was similar between Japanese patients and the overall population.

Among all Japanese patients, longer OS was observed with avelumab + BSC vs BSC alone (median OS, 24.7 vs 18.7 months; HR, 0.81 [95\% CI, 0.41-1.58]). This occurred despite a higher proportion of Japanese patients receiving a subsequent anticancer drug therapy in the control arm (avelumab arm, 61.1\%; control arm, 81.1\%), including a PD-1 or PD-L1 inhibitor (38.9\% vs $67.6 \%$, respectively). Subsequent anticancer drug therapy was higher in Japanese patients than in the overall population (avelumab vs control in the overall population: any subsequent anticancer drug therapy, $42.3 \%$ vs $61.7 \%$; subsequent PD-1/PD-L1

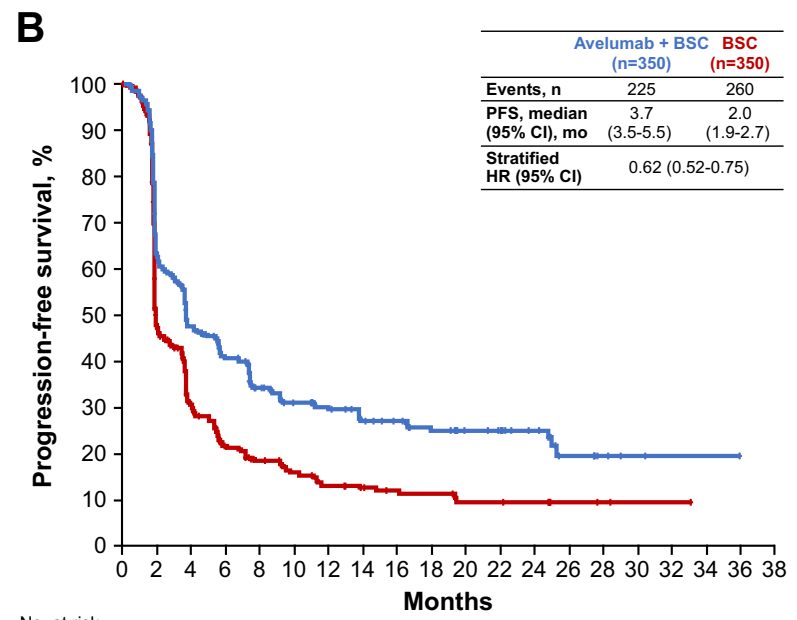
No. at risk

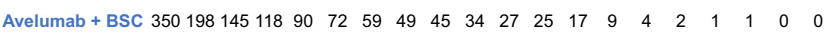

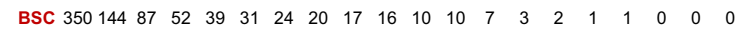

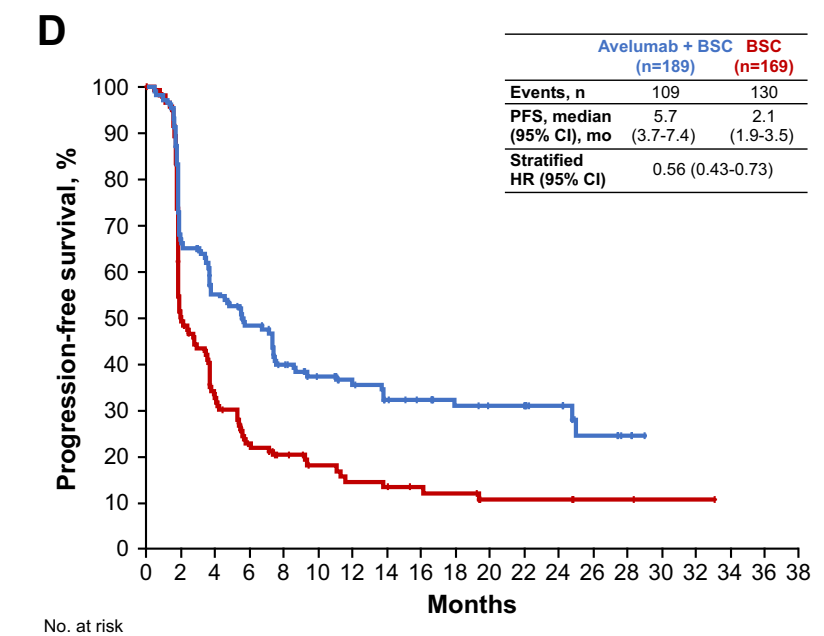

Avelumab + BSC $189 \begin{array}{lllllllllllllllllll}114 & 89 & 73 & 55 & 45 & 35 & 29 & 26 & 20 & 17 & 17 & 12 & 7 & 2 & 0 & 0 & 0 & 0 & 0\end{array}$ BSC $169 \begin{array}{lllllllllllllllllll}80 & 51 & 28 & 21 & 16 & 13 & 12 & 10 & 9 & 5 & 5 & 5 & 2 & 2 & 1 & 1 & 0 & 0 & 0\end{array}$

grammed death ligand 1, PFS progression-free survival. B and D From Powles et al. [13]. Copyright (C) 2020 Massachusetts Medical Society. Reprinted with permission

inhibitor, $6.3 \%$ vs $43.7 \%$ ), which may have led to longer OS in the Japanese subgroup. These data show that subsequent PD-1/PD-L1 inhibitor use after avelumab was also higher in Japanese patients than in the overall population. In Japanese patients with PD-L1 + tumors, median OS was similar in both arms; however, $95 \%$ CIs were wide because of the small numbers of patients and events. In addition, some differences were observed in demographic and disease characteristics between the treatment arms in Japanese patients with PD-L1 + tumors, which may have affected OS outcomes. Longer PFS was observed in the avelumab arm vs the control arm among Japanese patients, both in the overall subgroup (median, 5.6 vs 1.9 months; HR, 0.63 [95\% CI, 
Table 3 Confirmed objective response per BICR in the Japanese subgroup and the overall population. Source: Data for the overall population have been published previously in Ref. [13]. Copyright @ 2020 Massachusetts Medical Society. Reprinted with permission

\begin{tabular}{|c|c|c|c|c|}
\hline & \multicolumn{2}{|c|}{ Japanese subgroup $(n=73)$} & \multicolumn{2}{|c|}{ Overall population $(N=700)[13]$} \\
\hline & $\begin{array}{l}\text { Avelumab + BSC } \\
(n=36)\end{array}$ & $\operatorname{BSC}(n=37)$ & $\begin{array}{l}\text { Avelumab + BSC } \\
(n=350)\end{array}$ & $\begin{array}{l}\text { BSC } \\
(n=350)\end{array}$ \\
\hline \multicolumn{5}{|l|}{ Confirmed best overall response, $n(\%)$} \\
\hline Complete response & $1(2.8)$ & 0 & $21(6.0)$ & $3(0.9)$ \\
\hline Partial response & $1(2.8)$ & 0 & $13(3.7)$ & $2(0.6)$ \\
\hline Stable disease & $3(8.3)$ & $6(16.2)$ & $44(12.6)$ & $46(13.1)$ \\
\hline Noncomplete response/nonprogressive disease & $7(19.4)$ & $4(10.8)$ & $66(18.9)$ & $45(12.9)$ \\
\hline Progressive disease & $17(47.2)$ & $17(45.9)$ & $130(37.1)$ & $169(48.3)$ \\
\hline Not evaluable & $7(19.4)$ & $10(27.0)$ & $76(21.7)$ & $85(24.3)$ \\
\hline Objective response, $n(\%)$ & $2(5.6)$ & 0 & $34(9.7)$ & $5(1.4)$ \\
\hline$(95 \% \mathrm{CI})$ & $(0.7-18.7)$ & $(0-9.5)$ & $(6.8-13.3)$ & $(0.5-3.3)$ \\
\hline Disease control, $n(\%)$ & $12(33.3)$ & $10(27.0)$ & $144(41.1)$ & $96(27.4)$ \\
\hline$(95 \% \mathrm{CI})$ & $(18.6-51.0)$ & $(13.8-44.1)$ & $(35.9-46.5)$ & $(22.8-32.4)$ \\
\hline
\end{tabular}

$B I C R$ blinded independent central review, $B S C$ best supportive care

Table 4 Subsequent anticancer drug therapy in the Japanese subgroup and the overall population. Source: Data for the overall population have been published previously in Ref. [13]. Copyright ( 2020 Massachusetts Medical Society. Reprinted with permission

\begin{tabular}{|c|c|c|c|c|c|c|c|c|}
\hline & \multicolumn{4}{|c|}{ Japanese subgroup } & \multicolumn{4}{|c|}{ Overall population [13] } \\
\hline & \multicolumn{2}{|c|}{$\begin{array}{l}\text { Randomised patients } \\
(n=73)\end{array}$} & \multicolumn{2}{|c|}{$\begin{array}{l}\text { PD-L1 + population } \\
(n=42)\end{array}$} & \multicolumn{2}{|c|}{$\begin{array}{l}\text { Randomised patients } \\
(N=700)\end{array}$} & \multicolumn{2}{|c|}{$\begin{array}{l}\text { PD-L1 + population } \\
(N=358)\end{array}$} \\
\hline & $\begin{array}{l}\text { Avelumab } \\
+\mathrm{BSC} \\
(n=36)\end{array}$ & $\begin{array}{l}\mathrm{BSC}( \\
n=37)\end{array}$ & $\begin{array}{l}\text { Avelumab } \\
+\mathrm{BSC} \\
(n=19)\end{array}$ & $\begin{array}{l}\mathrm{BSC} \\
(n=23)\end{array}$ & $\begin{array}{l}\text { Avelumab } \\
+\mathrm{BSC} \\
(n=350)\end{array}$ & $\begin{array}{l}\mathrm{BSC} \\
(n=350)\end{array}$ & $\begin{array}{l}\text { Avelumab } \\
+ \text { BSC } \\
(n=189)\end{array}$ & $\begin{array}{l}\text { BSC } \\
(n=169)\end{array}$ \\
\hline $\begin{array}{l}\text { Discontinued } \\
\text { and received } \\
\text { subsequent drug } \\
\text { therapy, } n(\%)^{\mathrm{a}}\end{array}$ & $22(61.1)$ & $30(81.1)$ & $9(47.4)$ & $19(82.6)$ & $148(42.3)$ & $216(61.7)$ & $68(36.0)$ & $109(64.5)$ \\
\hline $\begin{array}{l}\text { PD-L1/PD-1 } \\
\text { inhibitor }\end{array}$ & $14(38.9)$ & $25(67.6)$ & $6(31.6)$ & $16(69.6)$ & $22(6.3)$ & $153(43.7)$ & $10(5.3)$ & $81(47.9)$ \\
\hline $\begin{array}{l}\text { Fibroblast growth } \\
\text { factor receptor } \\
\text { inhibitor }\end{array}$ & $1(2.8)$ & 0 & $1(5.3)$ & 0 & $9(2.6)$ & $8(2.3)$ & $3(1.6)$ & $4(2.4)$ \\
\hline Any other drug & $19(52.8)$ & $20(54.1)$ & $8(42.1)$ & $10(43.5)$ & $140(40.0)$ & $119(34.0)$ & $67(35.4)$ & $57(33.7)$ \\
\hline $\begin{array}{l}\text { Discontinued with } \\
\text { no subsequent } \\
\text { drug therapy, } n \\
(\%)\end{array}$ & $7(19.4)$ & $5(13.5)$ & $5(26.3)$ & $2(8.7)$ & $117(33.4)$ & $108(30.9)$ & $63(33.3)$ & $47(27.8)$ \\
\hline $\begin{array}{l}\text { Study treatment } \\
\text { ongoing, } n(\%)\end{array}$ & $7(19.4)$ & $2(5.4)$ & $5(26.3)$ & $2(8.7)$ & $85(24.3)$ & $26(7.4)$ & $58(30.7)$ & $13(7.7)$ \\
\hline
\end{tabular}

$B S C$ best supportive care, $P D-1$ programmed death $1, P D-L 1$ programmed death ligand 1

${ }^{\text {a }}$ Some patients received $>1$ category of subsequent therapy 
Table 5 Safety summary in the Japanese subgroup and the overall population. Source: Data for the overall population have been published previously in Ref. [13]. Copyright @ 2020 Massachusetts Medical Society. Reprinted with permission

\begin{tabular}{|c|c|c|c|c|}
\hline \multirow[t]{2}{*}{$n(\%)$} & \multicolumn{2}{|l|}{$\begin{array}{l}\text { Japanese subgroup } \\
(n=73)\end{array}$} & \multicolumn{2}{|c|}{$\begin{array}{l}\text { Overall safety population } \\
(N=689)[13]\end{array}$} \\
\hline & $\begin{array}{l}\text { Avelumab + BSC } \\
(n=36)\end{array}$ & $\begin{array}{l}\mathrm{BSC} \\
(n=37)\end{array}$ & $\begin{array}{l}\text { Avelumab + BSC } \\
(n=344)\end{array}$ & $\begin{array}{l}\text { BSC } \\
(n=345)\end{array}$ \\
\hline Any TEAE & $36(100)$ & $21(56.8)$ & $337(98.0)$ & $268(77.7)$ \\
\hline Grade $\geq 3$ TEAE & $18(50.0)$ & $3(8.1)$ & $163(47.4)$ & $87(25.2)$ \\
\hline Treatment-related TEAE & $27(75.0)$ & 0 & $266(77.3)$ & $4(1.2)$ \\
\hline Grade $\geq 3$ treatment-related TEAE & $5(13.9)$ & 0 & $57(16.6)$ & 0 \\
\hline Serious TEAE & $9(25.0)$ & $2(5.4)$ & $96(27.9)$ & $69(20.0)$ \\
\hline Serious treatment-related TEAE & $5(13.9)$ & 0 & $31(9.0)$ & 0 \\
\hline TEAE leading to dose reduction of avelumab & 0 & - & $1(0.3)$ & - \\
\hline TEAE leading to interruption of avelumab & $16(44.4)$ & - & $140(40.7)$ & - \\
\hline TEAE leading to discontinuation of study drug & $4(11.1)$ & 0 & $41(11.9)$ & 0 \\
\hline Treatment-related TEAE leading to discontinuation of study drug & $4(11.1)$ & 0 & $33(9.6)$ & 0 \\
\hline TEAE leading to death & $1(2.8)$ & 0 & $4(1.2)$ & $24(7.0)$ \\
\hline Treatment-related TEAE leading to death & $1(2.8)$ & 0 & $1(0.3)$ & 0 \\
\hline Immune-related $\mathrm{AE}$ & $13(36.1)$ & $2(5.4)$ & $101(29.4)$ & $5(1.4)$ \\
\hline Immune-related $\mathrm{AE}$ leading to discontinuation of study drug & $1(2.8)$ & 0 & $19(5.5)$ & 0 \\
\hline Infusion-related reaction $^{\mathrm{a}}$ & $10(27.8)$ & 0 & $74(21.5)$ & 0 \\
\hline
\end{tabular}

$A E$ adverse event, $B S C$ best supportive care, $T E A E$ treatment-emergent adverse event

${ }^{a}$ Composite term including several prespecified preferred terms in addition to signs and symptoms of infusion-related reaction

0.36-1.11]) and in those with PD-L1 + tumors (median, 5.6 vs 1.9 months; HR, 0.62 [95\% CI, 0.30-1.30]).

The safety profile of avelumab in Japanese patients was consistent with that of the overall population, and no safety concerns specific to Japanese patients were identified. Slight differences were seen in the most common TEAEs of any grade in Japanese patients treated with avelumab compared with the overall population. However, because the median relative dose intensity of avelumab was similar between Japanese patients and the overall population $(87.8 \%$ vs $88.2 \%)$, these observations might be due to ethnic differences or sampling constraints in a smaller patient group. No notable differences in the occurrence of irAEs were observed between Japanese patients and the overall population (36.1\% vs $29.4 \%$ ), and rates of discontinuation due to TEAEs (11.1\% vs $11.9 \%$ ) or irAEs ( $2.8 \%$ vs $5.5 \%$ ) were low in both groups.

In conclusion, avelumab $1 \mathrm{~L}$ maintenance treatment showed a favorable benefit-risk balance in Japanese patients, similar to that in the overall population. These results support avelumab $1 \mathrm{~L}$ maintenance as a new standard of care in Japanese patients with advanced UC that has not progressed with $1 \mathrm{~L}$ platinum-containing chemotherapy. 
Table 6 Most common TEAEs (related or unrelated to study treatment) in the Japanese subgroup and the overall population. Source: Data for the overall population have been published previously

\begin{tabular}{|c|c|c|c|c|c|c|c|c|}
\hline \multirow[t]{3}{*}{ Events, $n(\%)$} & \multicolumn{4}{|c|}{ Japanese subgroup $(n=73)$} & \multicolumn{4}{|c|}{ Overall safety population $(N=689)[13]$} \\
\hline & \multicolumn{2}{|c|}{ Avelumab + BSC $(n=36)$} & \multicolumn{2}{|c|}{$\operatorname{BSC}(n=37)$} & \multicolumn{2}{|c|}{ Avelumab + BSC $(n=344)$} & \multicolumn{2}{|c|}{$\operatorname{BSC}(n=345)$} \\
\hline & Any grade & Grade $\geq 3$ & Any grade & Grade $\geq 3$ & Any grade & Grade $\geq 3$ & Any grade & Grade $\geq 3$ \\
\hline Any TEAE & $36(100)$ & $18(50.0)$ & $21(56.8)$ & $3(8.1)$ & $337(98.0)$ & $163(47.4)$ & $268(77.7)$ & $87(25.2)$ \\
\hline Pyrexia & $10(27.8)$ & 0 & 0 & 0 & $51(14.8)$ & $1(0.3)$ & $12(3.5)$ & 0 \\
\hline Anemia & $7(19.4)$ & $4(11.1)$ & $1(2.7)$ & $1(2.7)$ & $39(11.3)$ & $13(3.8)$ & $23(6.7)$ & $10(2.9)$ \\
\hline Nasopharyngitis & $7(19.4)$ & 0 & $5(13.5)$ & 0 & $26(7.6)$ & 0 & $13(3.8)$ & 0 \\
\hline Constipation & $6(16.7)$ & 0 & $3(8.1)$ & 0 & $56(16.3)$ & $2(0.6)$ & $31(9.0)$ & 0 \\
\hline Nausea & $6(16.7)$ & 0 & 0 & 0 & $54(15.7)$ & $1(0.3)$ & $22(6.4)$ & $2(0.6)$ \\
\hline Rash & $6(16.7)$ & 0 & 0 & 0 & 40 (11.6) & $1(0.3)$ & $4(1.2)$ & 0 \\
\hline Hypothyroidism & $6(16.7)$ & 0 & 0 & 0 & $40(11.6)$ & $1(0.3)$ & $2(0.6)$ & 0 \\
\hline Vomiting & $5(13.9)$ & 0 & $2(5.4)$ & 0 & $43(12.5)$ & $4(1.2)$ & $12(3.5)$ & $2(0.6)$ \\
\hline Hematuria & $5(13.9)$ & 0 & $1(2.7)$ & $1(2.7)$ & $36(10.5)$ & $6(1.7)$ & $37(10.7)$ & $5(1.4)$ \\
\hline Infusion-related reaction & $5(13.9)$ & 0 & 0 & 0 & $35(10.2)$ & $3(0.9)$ & 0 & 0 \\
\hline Cancer pain & $4(11.1)$ & $1(2.8)$ & $2(5.4)$ & 0 & $8(2.3)$ & $1(0.3)$ & $6(1.7)$ & $1(0.3)$ \\
\hline Back pain & $4(11.1)$ & 0 & $3(8.1)$ & 0 & $55(16.0)$ & $4(1.2)$ & $34(9.9)$ & $8(2.3)$ \\
\hline Diarrhea & $4(11.1)$ & 0 & 0 & 0 & $57(16.6)$ & $2(0.6)$ & $17(4.9)$ & $1(0.3)$ \\
\hline Arthralgia & $4(11.1)$ & 0 & 0 & 0 & $56(16.3)$ & $2(0.6)$ & $19(5.5)$ & 0 \\
\hline Headache & $4(11.1)$ & 0 & 0 & 0 & $24(7.0)$ & $1(0.3)$ & $9(2.6)$ & $1(0.3)$ \\
\hline Hyperthyroidism & $4(11.1)$ & 0 & 0 & 0 & $21(6.1)$ & 0 & $1(0.3)$ & 0 \\
\hline Stomatitis & $4(11.1)$ & 0 & $1(2.7)$ & 0 & $7(2.0)$ & 0 & $1(0.3)$ & 0 \\
\hline Dental caries & $4(11.1)$ & 0 & 0 & 0 & $4(1.2)$ & 0 & 0 & 0 \\
\hline WBC count decreased & $4(11.1)$ & 0 & 0 & 0 & $4(1.2)$ & 0 & 0 & 0 \\
\hline Pyelonephritis & $3(8.3)$ & $2(5.6)$ & $1(2.7)$ & 0 & $4(1.2)$ & $3(0.9)$ & $3(0.9)$ & $2(0.6)$ \\
\hline Urinary tract infection & $3(8.3)$ & $1(2.8)$ & $1(2.7)$ & 0 & $59(17.2)$ & $15(4.4)$ & $36(10.4)$ & $9(2.6)$ \\
\hline Pruritus & $3(8.3)$ & 0 & $1(2.7)$ & 0 & $59(17.2)$ & $1(0.3)$ & $6(1.7)$ & 0 \\
\hline Amylase increased & $2(5.6)$ & $2(5.6)$ & 0 & 0 & $23(6.7)$ & $12(3.5)$ & $3(0.9)$ & $2(0.6)$ \\
\hline Blood triglycerides increased & $2(5.6)$ & $2(5.6)$ & 0 & 0 & $3(0.9)$ & $3(0.9)$ & 0 & 0 \\
\hline Fatigue & $2(5.6)$ & $1(2.8)$ & $1(2.7)$ & 0 & $61(17.7)$ & $6(1.7)$ & $24(7.0)$ & $2(0.6)$ \\
\hline Cough & $2(5.6)$ & 0 & $2(5.4)$ & 0 & $44(12.8)$ & $1(0.3)$ & $16(4.6)$ & 0 \\
\hline Decreased appetite & $1(2.8)$ & 0 & $4(10.8)$ & 0 & $47(13.7)$ & $1(0.3)$ & $23(6.7)$ & $2(0.6)$ \\
\hline Asthenia & 0 & 0 & 0 & 0 & $56(16.3)$ & 0 & $19(5.5)$ & $4(1.2)$ \\
\hline
\end{tabular}

Table shows TEAEs (preferred terms) occurring at any grade in $\geq 10 \%$ or grade $\geq 3$ in $\geq 5 \%$ of patients in either arm in the Japanese subgroup or the overall population

$B S C$ best supportive care, TEAE treatment-emergent adverse event, $W B C$ white blood cell

Supplementary Information The online version contains supplementary material available at https://doi.org/10.1007/s10147-021-02067-8.

Acknowledgements The authors would like to thank the patients and their families, investigators, co-investigators, and the study teams at each of the participating centers as well as Hisanaga Ohashi and Katsushi Namazu of Pfizer R\&D Japan for data collection. This trial was sponsored by Pfizer as part of an alliance between Pfizer and Merck (CrossRef Funder ID: https://doi.org/10.13039/100009945). Study drug was provided by Pfizer and Merck. Pfizer collected and analyzed the data and contributed to the interpretation of the study in conjunction with investigators. All authors had access to study data and had final responsibility for the decision to submit for publication. Medical writing support was provided by Felicia Barklund of ClinicalThinking and funded by Pfizer and Merck. in Ref. [13]. Copyright (C) 2020 Massachusetts Medical Society. Reprinted with permission 


\section{Declarations}

Conflict of interest Y. Tomita has received honoraria from Astellas Pharma, Bristol Myers Squibb, Chugai Pharma, Novartis, Ono Pharmaceutical, and Pfizer; served in a consulting or advisory role for Novartis, Ono Pharmaceutical, and Taiho Pharmaceutical; and received research funding from Astellas Pharma, AstraZeneca, Chugai Pharma, Eisai, MSD, Novartis, Ono Pharmaceutical, Pfizer, and Takeda. Y. Yamamoto reports no disclosures. N. Tsuchiya has received honoraria from Astellas Pharma, AstraZeneca, Bristol Myers Squibb, Janssen, Merck \& Co., MSD, Novartis, Pfizer, Sanofi, and Takeda and received research funding from Pfizer and Takeda. H. Kanayama has received research funding from Astellas Pharma, Fujifilm, Kissei Pharmaceutical, MSD KK, Nihon Medi-Physics, Ono Pharmaceutical, Taiho Pharmaceutical, and Takeda. M. Eto has served in a consulting or advisory role for AstraZeneca, Bristol Myers Squibb, Chugai Pharma, Eisai, Johnson \& Johnson, Merck, Olympas, Ono Pharmaceutical, and Pfizer; has provided speakers services for Bristol Myers Squibb, Chugai Pharma, Janssen, MSD, Novartis, Ono Pharmaceutical, Pfizer, and Takeda; and received research funding from Astellas Pharma, Bayer, Kissei Pharmaceutical, Ono Pharmaceutical, Sanofi, and Takeda. $\mathrm{H}$. Miyake has received honoraria and research funding from and served in a consulting or advisory role for Pfizer. T. Powles has received honoraria and research funding from and served in a consulting or advisory role for Astellas Pharma, AstraZeneca, Bristol Myers Squibb, Eisai, EMD Serono Research \& Development Institute, Inc., Billerica, MA, USA, an affiliate of Merck KGaA, Exelixis, Ipsen, Johnson \& Johnson, Merck \& Co., MSD, Novartis, Pfizer, Seattle Genetics, and Roche; has received honoraria from and served in a consulting or advisory role for Incyte; and has received travel and accommodation expenses from AstraZeneca, Ipsen, MSD, Pfizer, and Roche. M. Yoshida reports employment at Pfizer R\&D Japan. Y. Koide reports employment at Pfizer R\&D Japan and owns Pfizer stock. Y. Umeyama reports employment at Pfizer R\&D Japan and owns Pfizer stock. A. di Pietro reports employment at Pfizer and owns Pfizer stock. H. Uemura has provided speaker services for Bayer, Bristol Myers Squibb, Janssen, MSD, and Pfizer and has received research funding from Astellas Pharma, AstraZeneca, Daiichi Sankyo, Kissei Pharmaceutical, Ono Pharmaceutical, Sanofi, and Takeda.

Open Access This article is licensed under a Creative Commons Attribution 4.0 International License, which permits use, sharing, adaptation, distribution and reproduction in any medium or format, as long as you give appropriate credit to the original author(s) and the source, provide a link to the Creative Commons licence, and indicate if changes were made. The images or other third party material in this article are included in the article's Creative Commons licence, unless indicated otherwise in a credit line to the material. If material is not included in the article's Creative Commons licence and your intended use is not permitted by statutory regulation or exceeds the permitted use, you will need to obtain permission directly from the copyright holder. To view a copy of this licence, visit http://creativecommons.org/licenses/by/4.0/.

\section{References}

1. Matsuda T, Okuyama A (2017) Incidence rate for bladder cancer in Japanese in Japan and in the United States from the cancer incidence in five continents. Jpn J Clin Oncol 47(3):284-285. https://doi.org/10.1093/jjco/hyx017

2. Bray F, Ferlay J, Soerjomataram I et al (2018) Global cancer statistics 2018: GLOBOCAN estimates of incidence and mortality worldwide for 36 cancers in 185 countries. CA Cancer J Clin 68(6):394-424. https://doi.org/10.3322/caac.21492
3. National Comprehensive Cancer Network NCCN Clinical Practice Guidelines. Bladder Cancer. V4.2021. https://www.nccn.org/profe ssionals/physician_gls/pdf/bladder.pdf. Accessed 19 Aug 2021

4. Matsumoto H, Shiraishi K, Azuma H et al (2020) Clinical Practice Guidelines for Bladder Cancer 2019 update by the Japanese Urological Association: summary of the revision. Int J Urol 27(9):702-709. https://doi.org/10.1111/iju.14281

5. De Santis M, Bellmunt J, Mead G et al (2012) Randomized phase II/III trial assessing gemcitabine/carboplatin and methotrexate/ carboplatin/vinblastine in patients with advanced urothelial cancer who are unfit for cisplatin-based chemotherapy: EORTC study 30986. J Clin Oncol 30(2):191-199. https://doi.org/10.1200/JCO. 2011.37.3571

6. Dogliotti L, Carteni G, Siena S et al (2007) Gemcitabine plus cisplatin versus gemcitabine plus carboplatin as first-line chemotherapy in advanced transitional cell carcinoma of the urothelium: results of a randomized phase 2 trial. Eur Urol 52(1):134-141. https://doi.org/10.1016/j.eururo.2006.12.029

7. von der Maase H, Sengelov L, Roberts JT et al (2005) Long-term survival results of a randomized trial comparing gemcitabine plus cisplatin, with methotrexate, vinblastine, doxorubicin, plus cisplatin in patients with bladder cancer. J Clin Oncol 23(21):46024608. https://doi.org/10.1200/JCO.2005.07.757

8. Kim TJ, Cho KS, Koo KC (2020) Current status and future perspectives of immunotherapy for locally advanced or metastatic urothelial carcinoma: a comprehensive review. Cancers (Basel) 12(1):192. https://doi.org/10.3390/cancers 12010192

9. Collins JM, Gulley JL (2019) Product review: avelumab, an antiPD-L1 antibody. Hum Vaccin Immunother 15(4):891-908. https:// doi.org/10.1080/21645515.2018.1551671

10. Grenga I, Donahue RN, Lepone LM et al (2016) A fully human IgG1 anti-PD-L1 MAb in an in vitro assay enhances antigen-specific T-cell responses. Clin Transl Immunology 5(5):e83. https:// doi.org/10.1038/cti.2016.27

11. Boyerinas B, Jochems C, Fantini M et al (2015) Antibody-dependent cellular cytotoxicity activity of a novel anti-PD-L1 antibody avelumab (MSB0010718C) on human tumor cells. Cancer Immunol Res 3(10):1148-1157. https://doi.org/10.1158/2326-6066. CIR-15-0059

12. Uemura M, Tomita Y, Miyake H et al (2020) Avelumab plus axitinib vs sunitinib for advanced renal cell carcinoma: Japanese subgroup analysis from JAVELIN Renal 101. Cancer Sci 111(3):907-923. https://doi.org/10.1111/cas.14294

13. Powles T, Park SH, Voog E et al (2020) Avelumab maintenance therapy for advanced or metastatic urothelial carcinoma. N Engl J Med 383(13):1218-1230. https://doi.org/10.1056/NEJMoa2002 788

14. European Society for Medical Oncology (2020) eUpdate-bladder cancer treatment recommendations (July 16, 2020). https://www. esmo.org/guidelines/genitourinary-cancers/bladder-cancer/eupda te-bladder-cancer-treatment-recommendations4. Accessed 19 Aug 2021

15. Witjes JA, Bruins HM, Cathomas R et al (2021) European Association of Urology guidelines on muscle-invasive and metastatic bladder cancer. https://uroweb.org/guideline/bladder-cancer-muscle-invasive-and-metastatic/. Accessed 19 Aug 2021

16. Japanese Urological Association. Supplement to clinical practice guidelines for bladder cancer. https://www.urol.or.jp/lib/ files/other/guideline/39_bladder_cancer_2019_rev2021_info. pdf. Accessed 1 Dec $202 \overline{1}$

Publisher's Note Springer Nature remains neutral with regard to jurisdictional claims in published maps and institutional affiliations. 


\section{Authors and Affiliations}

\section{Yoshihiko Tomita $^{1} \cdot$ Yoshiaki Yamamoto $^{2} \cdot$ Norihiko Tsuchiya $^{3} \cdot$ Hiroomi Kanayama $^{4} \cdot$ Masatoshi Eto $^{5}$.} Hideaki Miyake $^{6} \cdot$ Thomas Powles $^{7} \cdot$ Mizuki Yoshida $^{8} \cdot$ Yuichiro Koide $^{8} \cdot$ Yoshiko Umeyama $^{8} \cdot$ Alessandra di Pietro $^{9}$. Hirotsugu Uemura ${ }^{10}$

1 Department of Urology and Department of Molecular Oncology, Niigata University Graduate School of Medicine, 1-757 Asahimachi, Chuou-ku, Niigata 951-8510, Japan

2 Department of Urology, Graduate School of Medicine, Yamaguchi University, 1-1-1 Minami-Kogushi, Ube, Yamaguchi 755-8505, Japan

3 Department of Urology, Yamagata University Faculty of Medicine, 2-2-2 Iida-Nishi, Yamagata 990-9585, Japan

4 Department of Urology, Tokushima University Graduate School of Biomedical Sciences, 3-18-15, Kuramoto-cho, Tokushima 770-8503, Japan

5 Department of Urology, Kyushu University Graduate School of Medical Sciences, 3-1-1 Maidashi, Higashi-ku, Fukuoka 812-8582, Japan
6 Department of Urology, Hamamatsu University School of Medicine, 1-20-1 Handayama, Higashi-ku, Hamamatsu, Shizuoka 431-3192, Japan

7 Experimental Cancer Medicine Centre, Barts Cancer Institute, Queen Mary University of London, St Bartholomew's Hospital, West Smithfield, London EC1A 7BE, UK

8 Pfizer R\&D Japan, 3-22-7 Yoyogi, Shibuya-ku, Tokyo 151-8589, Japan

9 Pfizer srl, Via Anna Maria Mozzoni, 12, 20152 Milan, Italy

10 Department of Urology, Faculty of Medicine, Kindai University, 377-2 Ohno-Higashi, Osakasayama, Osaka 589-8511, Japan 